Jiménez-Hernández, D., Sancho-Requena, P. \& Sánchez-Fuentes, S. (2021). Estudio acerca de las opiniones del profesorado universitario en la Región de Murcia sobre la formación de métodos activos. Revista Electrónica Interuniversitaria de Formación del Profesorado, 24(2), 113-124.

\title{
Estudio acerca de las opiniones del profesorado universitario en la Región de Murcia sobre la formación de métodos activos
}

\author{
David Jiménez Hernández ${ }^{(1)}$, Patricia Sancho Requena ${ }^{(2)}$, Sergio Sánchez Fuentes ${ }^{(3)}$ \\ ${ }^{(1)}$ Universidad Miguel Hernández, ${ }^{(2)}$ Universidad de Valencia, ${ }^{(3)}$ Universidad Autónoma de \\ Madrid
}

\section{Resumen}

La formación del profesorado universitario supone el principal vehículo de cambio y mejora educativa en el marco del Espacio Europeo de Educación Superior (EEES). Sin embargo, existen una serie de factores que pueden impedir su formación y, por tanto, su repercusión en los estudiantes y en la sociedad. En este sentido, este trabajo describe las posibles dificultades que pueden surgir respecto de variables tales como: los años de experiencia docente, edad, género, área del conocimiento en la que imparten docencia, figura profesional o formación universitaria recibida. Para ello, se desarrolló un cuestionario ad hoc que fue aplicado a 501 docentes universitarios. Los resultados mostraron que la falta de tiempo y de ofertas formativas cercanas suponen los principales impedimentos para una mejor formación. No obstante, llevar poco tiempo como docente o preferir otro tipo de formación, mostraron también significatividad dentro de las variables estudiadas. De esta manera, los autores de este trabajo concluyen que se deberían ampliar los incentivos para que los docentes estén motivados con el fin de mejorar su calidad docente a través de nuevas metodologías activas, además de establecer planes sólidos en la oferta de formación académica de las universidades.

\section{Palabras clave}

Formación; Educación Superior; Profesorado, Aprendizaje activo.

\section{Contacto:}

David Jiménez Hernández, djimenez@umh.es, Departamento de Estadística, Matemáticas e Informática, Avenida Universidad s/n, 03202 Elche (España). 


\title{
Study on the opinions of university teachers in the Region of Murcia on the training of active methods
}

\begin{abstract}
The training of university professors is the principal way to change and to improve the education in the framework of the European Higher Education Area (EHEA). However, there are several factors that can impede their training and, therefore, their impact on students and society. In this sense, this paper describes that possible difficulties may arise regarding variables such as: years of teaching experience, age, gender, area of knowledge in which they teach, professional figure or university training received. For this, an ad hoc questionnaire was developed that was applied to 501 university professors. The results showed how the lack of time and nearby training offers are the main impediments to better training. Despite having been a teacher for a short time or preferring another type of training, they also showed significance within the variables studied. In this way, the authors of this work conclude that incentives should be expanded so that teachers are motivated in order to improve their teaching quality through new active methodologies, in addition to establishing solid plans in the offer of academic training of the universities.
\end{abstract}

\section{Key words}

Training; Higher Education; Professors; Active Learning.

\section{Introducción}

La formación del profesorado es imprescindible para atender al cambio (Escudero, 2020) y, por lo tanto, es la herramienta necesaria para poder atender a las nuevas formas de aprender que demandan las sociedades actuales. Tal y como explica García (2020), ya desde 1995, la Nacional Staff Development Council corroboró que el desarrollo profesional no afecta únicamente a los docentes, sino que afecta a toda la institución y debe ser un compromiso de todos. Por esta razón, las actuaciones de mejora no deben focalizarse únicamente en la práctica profesional, sino que debe afectar a todos los aspectos y a todo el personal de la institución universitaria.

La investigación que se ha desarrollado sobre la formación del profesorado es un campo joven en comparación con la investigación en la enseñanza. Aunque la investigación en la formación del profesorado tiene sus raíces en la anterior, este tipo de investigación se ha desarrollado de forma aislada con respecto a la segunda (Monereo, 2013). Una mayor conexión de los campos de la enseñanza con la investigación en las organizaciones y la implementación de políticas llevaría a seguir avanzado en la compresión de la enseñanza como práctica y preparación de los docentes (Vargas y Arce, 2020). En este sentido, Marcelo (2006) expone que la formación también es un problema al necesitar que el docente universitario esté motivado, además de encontrar la fórmula para implicar a éstos en la búsqueda de su excelencia a través de múltiples estrategias o metodologías activas que favorezcan el aprendizaje del alumnado. De la misma manera, siempre que se instauran reformas educativas, la formación es el mejor instrumento para favorecer los procesos de cambio (Domingo-Coscollola, Bosco, Carrasco Segovia y Sánchez Valero, 2020). Así, desde el cambio propuesto por el Espacio Europeo de Educación Superior (EEES) diversos estudios 
sobre la formación del profesorado universitario arrojan luz acerca de la importancia para lograr transformaciones educativas a través de la formación holística de sus profesionales (Rabadán y Hernández, 2012).

Por otro lado, González, Macías, Rodríguez y Aguilera (2009) atribuyen que el cambio metodológico que debe asumir el sistema universitario a raíz del EEES, supone un camino hacia una perspectiva más activa y centrada en el alumnado. Gallardo (2010) añade que el cambio de paradigma hacia un modelo basado en el "aprende a aprender" implica además un cambio del sistema didáctico, con una clara apuesta por métodos y estilos de aprendizaje activos. Además, en trabajos más recientes, Jiménez (2018) apunta que se debería desarrollar desde el propio sistema universitario un modelo que tenga en cuenta de manera más activa al propio alumnado. Aunque el aprendizaje sea un proceso destinado al estudiante, éste está condicionado por múltiples variables que el profesorado puede modificar (Yerovi y Riascos, 2019). Resulta obvio entonces que la actuación didáctica del docente en el proceso de aprendizaje constituye un factor principal en el alumnado.

Por otro lado, tal y como describen Arribas, Carabias y Monreal (2010), un aspecto importante que se debe destacar es la reflexión acerca de tres aspectos fundamentales. En primer lugar, es importante señalar el desarrollo de competencias docentes y profesionales como eje fundamental de la mejora didáctica; en segundo lugar, la utilización de metodologías activas como medio de renovación de la práctica docente universitaria tradicional supone una reconceptualización de un modelo anclado en el siglo anterior; y, en tercer lugar, la implementación de un sistema de evaluación formativo como vía para actualizar los conocimientos didácticos y pedagógicos de una buena parte del profesorado universitario. Además, autores como Triadó, Estebanell, Márquez y del Corral (2014), consideraron indispensables aquellas competencias relacionadas con las dichas metodologías ligadas a la innovación. Por este motivo, para que puedan desarrollar competencias sobre el conocimiento, selección, implementación y evaluación de los diferentes métodos, es necesario que dichos docentes tengan acceso a una formación específica y de calidad en métodos actuales (Ponce, Gómez, Kruscthalia y Viteri, 2019). Además, y como ya consideraba el Ministerio de Educación y Ciencia (MEC, 2006), cualquier renovación metodológica no va a ser posible sin una formación del profesorado, que dé lugar a un cambio que pase de la lección magistral como método predominante para recurrir a otras metodologías donde se fomente la participación activa del alumnado (Rabadán y Hernández, 2012). Así, Jiménez, González y Tornel (2018), demostraron que cuando al profesorado recibe formación sobre métodos activos, éstos responden con una mayor utilización de los mismos en las aulas.

Las políticas de formación presentan diferentes compromisos que van desde aspectos más teóricos, a otros más prácticos para facilitar el desarrollo de la profesionalidad docente (Escudero y Portela, 2015). Para Caballero y Bolívar (2015), la investigación en el campo de la formación docente supone un pilar fundamental para que la sociedad de la información evolucione de manera adecuada. Esta afirmación, además, se completa con lo que expone Hazelkorn (2015) respecto de la necesidad de impulsar aquellos méritos ligados a la obtención de una formación pedagógica. Y es que, desgraciadamente la capacitación de los docentes tiene un peso menor del deseado (Rodríguez, 2020) en la carrera profesional de los profesores universitarios. No obstante, la Comisión Europea (2017) señaló que la relevancia de la formación pedagógica de los docentes universitarios supone un elemento vital para una enseñanza de calidad.

Por todo ello, este trabajo tiene como objetivo principal estudiar cuáles son los principales inconvenientes que obstaculizan al profesorado universitario de la Región de Murcia la formación en métodos activos, para de este modo, poder proponer planes de formación 
adecuados con el fin de mejorar los procesos de enseñanza y aprendizaje en la educación superior de la región, y por extensión, del resto del país.

\section{Metodología}

\section{Diseño y participantes}

El diseño de investigación empleado fue, ex post facto, prospectivo de grupo único (Montero y León, 2007) utilizando un cuestionario de manera transversal.

Para ello, se contó con una muestra representativa de 501 docentes universitario de la Región de Murcia (España), siendo la muestra total de 3192, distribuidas entre las tres universidades que forman parte de ésta, siendo un $49.50 \%$ de la Universidad de Murcia, el $41.71 \%$ de la Universidad Católica San Antonio de Murcia y un $8.78 \%$ de la Universidad Politécnica de Cartagena. La media de edad fue de 42.98 años ( $D T=9,785$ ), correspondiendo un $54.5 \%$ de hombres y un $46.49 \%$ de mujeres. Así mismo, la media de años de experiencia docente ascendía a $13 \cdot 3$ años ( $D T=10,03)$.

\section{Instrumento}

El instrumento empleado para extraer la información fue una encuesta diseñada ad hoc, compuesta por un ítem con cuatros impedimentos con una escala tipo Likert, una pregunta abierta y, una última cuestión dicotómica (sí o no), sobre si estarían dispuesto a seguir formándose en estos métodos. Para seleccionar los ítems de la encuesta, se empleó el Método Delphi con expertos universitarios de diferentes ramas educativas (Jiménez, Tornel y González, 2015).

\section{Diseño y participantes}

Para alcanzar los objetivos planteados en la investigación, en primer lugar, se calcularon estadísticos descriptivos. Además, se llevaron a cabo cálculos relativos a estadística inferencial más concretamente Pruebas $t$, ANOVAs y correlaciones de Pearson. Estas últimas se interpretaron siguiendo las especificaciones de Cohen (1988): de pequeño tamaño cuando $r=.10$, moderadas cuando $r=.30$ y elevadas cuando $r=.50$ o superior.

Por último, se incluyó el cálculo y evaluación de tamaños del efecto (eta-cuadrado) basándose en las guías de Cohen (1992) para interpretar la magnitud de dichos efectos: 02 como efecto bajo, .13 como efecto moderado y .26 como efecto elevado. Todos los análisis se realizaron con SPSS 26.

\section{Resultados}

Desde la perspectiva de la formación en métodos activos, en el primer ítem de la encuesta, se pregunta por los "impedimentos que el profesorado haya podido tener para formarse en métodos activos" (Figura 1). En primer lugar, los docentes presentan una media de 3.25 (DT = 1.32) en la opción "falta de tiempo", 2.69 (DT = 1.34) en "falta de ofertas educativas cercanas", 2.37 (DT = 1.32) en "considerar más importante otro tipo de formación" y 1.97 (DT = 1.33) al pensar que llevan "poco tiempo como docente".

Además, en la pregunta abierta sobre impedimentos, los propios participantes añaden otros aspectos tales como: el elevado coste económico, falta de calidad de estos, falta de información sobre los cursos que se realizan, escasa oferta a distancia o telemática, coincidencia del horario de los cursos con otras obligaciones y tener que dedicar mucho tiempo a la investigación. 


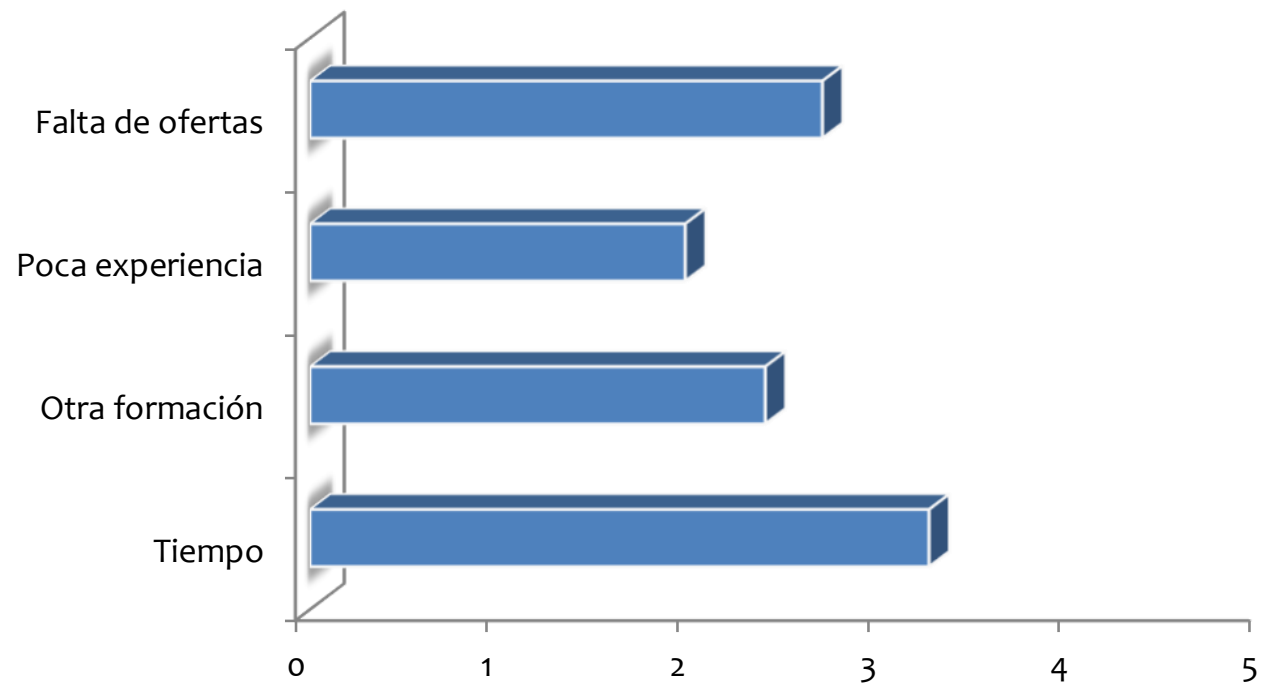

Figura 1. Impedimentos para formarse en métodos activos

Nota: Tiempo= Falta de tiempo; Otra formación= He considerado más importante otro tipo de formación; Poca experiencia = Llevo poco tiempo como docente; Falta de ofertas= Falta de ofertas educativas cercanas.

Pese a dichos impedimentos, en el segundo ítem, en el que se preguntó a los docentes si, "estaría dispuesto a seguir formándose en métodos activos", el $87.8 \%$ respondió afirmativamente. Profundizando en el estudio de los impedimentos, todos presentan correlaciones estadísticamente significativas entre ellos (Tabla 1). De igual modo, se hallaron correlaciones estadísticamente significativas entre todos éstos con la edad y los años de experiencia, a excepción de "he considerado más importante otro tipo de formación" (Tabla 1).

Tabla 1.

Correlaciones entre tipología de impedimentos, edad y años de experiencia

\begin{tabular}{lllllll}
\hline & Tiempo & Formación & Docente & Ofertas & Edad & Experiencia \\
\hline Tiempo & 1 & - & - & - & - & - \\
Formación & $.29^{* *}$ & 1 & - & - & - & - \\
Docente & $.10^{*}$ & $.10^{*}$ & 1 & - & - & - \\
Ofertas & $.24^{* *}$ & $.18^{* *}$ & $.38^{* *}$ & 1 & - & - \\
Edad & $-.10^{*}$ & .049 & $-.30^{* *}$ & $-.17^{* *}$ & 1 & - \\
Experiencia & $-.09^{*}$ & .065 & $-.49^{* *}$ & $-.25^{* *}$ & $.77^{* *}$ & 1 \\
\hline
\end{tabular}

Nota: ${ }^{*} p<.05 ; * p<.01$. Tiempo = Falta de tiempo; Formación = He considerado más importante otro tipo de formación; Docente = Llevo poco tiempo como docente; Ofertas = Falta de ofertas educativas cercanas; Experiencia $=$ años de experiencia docente 
Relacionando los impedimentos con el género, las Pruebas $t$ arrojaron un resultado estadísticamente significativo únicamente en el caso de, "he considerado más importante otro tipo de formación" ( $\left.t_{493,571}=-3.731, p<.01\right)$, con una media de $2.58(D T=1.22)$ en el caso de profesores y $2.14(D T=1.38)$ en el de las profesoras. En el resto de los impedimentos, los resultados no fueron estadísticamente significativos (Falta de tiempo: $t_{496}=-3.731, p>.05$; "Llevo poco tiempo como docente": $t_{494}=.944, p>.05$; "Falta de ofertas formativas cercanas": $\left.t_{494}=1.284, p>.05\right)$.

Con respecto a la rama de conocimiento, tal y como se observa en la Tabla 2, los ANOVAs mostraron resultados estadísticamente significativos en el caso de, "llevo poco tiempo como docente", revelando las pruebas a posteriori diferencias estadísticamente significativas entre Ciencias Sociales y Jurídicas y Artes y Humanidades, Ciencias Sociales y Jurídicas y Ciencias, y esta última y Ciencias de la Salud (Tabla 3).

Tabla 2.

ANOVAs entre impedimentos y rama de conocimiento, figura contractual, formación y formación posterior

\begin{tabular}{lllllll}
\hline & Impedimento & $F$ & $g l$ & Error & $p$ & $\mathrm{~h}^{2}$ \\
\hline Rama de & Tiempo & .484 & 4 & 482 & .748 & .004 \\
conocimiento & Formación & 2.347 & 4 & 482 & .054 & .019 \\
& Docente & 3.848 & 4 & 482 & .004 & .031 \\
& Ofertas & .675 & 4 & 482 & .610 & .006 \\
Figura & Tiempo & .815 & 7 & 419 & .576 & .017 \\
Contractual & Formación & 1.674 & 7 & 419 & .114 & .028 \\
& Docente & 15.160 & 7 & 419 & .000 & .195 \\
& Ofertas & 4.604 & 7 & 419 & .000 & .075 \\
Formación & Tiempo & .212 & 3 & 494 & .888 & .001 \\
& Formación & .902 & 3 & 494 & .440 & .005 \\
& Docente & 3.714 & 3 & 494 & .012 & .022 \\
Formación & Ofertas & 4.180 & 3 & 494 & .006 & .025 \\
posterior & Tiempo & 1.109 & 2 & 493 & .331 & .004 \\
& Formación & 2.919 & 2 & 493 & .055 & .012 \\
& Docente & 20.950 & 2 & 493 & .000 & .078 \\
& Ofertas & 3.520 & 2 & 493 & .030 & .014 \\
\hline
\end{tabular}

Nota: Tiempo = Falta de tiempo; Formación = He considerado más importante otro tipo de formación; Docente $=$ Llevo poco tiempo como docente; Ofertas = Falta de ofertas educativas cercanas . 
Tabla 3.

Estadísticos descriptivos en impedimentos según rama de conocimiento

\begin{tabular}{llllllllll}
\hline & \multicolumn{2}{l}{ Tiempo } & \multicolumn{3}{c}{ Formación } & \multicolumn{2}{c}{ Docente } & \multicolumn{2}{c}{ Ofertas } \\
& $M$ & DT & $M$ & DT & $M$ & DT & $M$ & DT \\
\hline Artes y humanidades & 3.15 & 1.32 & 2.12 & 1.24 & 1.45 & .93 & 2.70 & 1.21 \\
Ciencias & 3.12 & 1.38 & 2.74 & 1.36 & 1.50 & 1.04 & 2.40 & 1.27 \\
Ciencias de la salud & 3.38 & 1.50 & 2.24 & 1.26 & 2.06 & 1.42 & 2.72 & 1.37 \\
Ciencias sociales y jurídicas & 3.28 & 1.39 & 2.36 & 1.30 & 2.17 & 1.36 & 2.78 & 1.34 \\
Ingeniería y arquitectura & 3.17 & 1.52 & 2.67 & 1.48 & 1.88 & 1.29 & 2.74 & 1.32 \\
\hline
\end{tabular}

Nota: Tiempo = Falta de tiempo; Formación = He considerado más importante otro tipo de formación; Docente $=$ Llevo poco tiempo como docente; Ofertas = Falta de ofertas educativas cercanas.

En el caso de la figura contractual, se obtuvieron correlaciones estadísticamente significativas en los impedimentos $B\left(r_{x y}=.100, p<.05\right), C\left(r_{x y}=-.428, p<.01\right)$ y $D\left(r_{x y}=-.259, p\right.$ $<$.01). Para indagar más al respecto de estas diferencias, se calcularon ANOVAs, obteniéndose resultados estadísticamente significativos en el caso de, "llevo poco tiempo como docente" y "falta de ofertas formativas cercanas". En ambos casos, las pruebas a posteriori muestras diferencias estadísticamente significativas entre Asociados y Contratados Doctores $(p<.05)$, Titulares $(p<.1)$ y Catedráticos $(p<.01)$. Pero, además, en el caso de "falta de ofertas formativas cercanas", se observan diferencias estadísticamente significativas entre Titulares y Catedráticos cuando se comparan con Ayudantes $(p<.05)$, Ayudantes Doctores $(p<.01)$ y Contratados Doctores $(p<.01)$. Se pueden observar las medias por figura contractual en la Tabla 4.

Tabla 4.

Estadísticos descriptivos en impedimentos según Figura Contractual

\begin{tabular}{|c|c|c|c|c|c|c|c|c|c|}
\hline & & \multicolumn{2}{|c|}{ Tiempo } & \multicolumn{2}{|c|}{ Formación } & \multicolumn{2}{|c|}{ Docente } & \multicolumn{2}{|c|}{ Ofertas } \\
\hline & & $M$ & DT & $M$ & DT & $M$ & DT & $M$ & DT \\
\hline Becario & & 2.75 & 2.062 & 2.25 & 1.500 & 4.00 & 2.000 & 3.00 & 1.414 \\
\hline Asociado & & 3.12 & 1.468 & 2.13 & 1.227 & 2.55 & 1.446 & 3.24 & 1.414 \\
\hline $\begin{array}{l}\text { Ayudante } \\
\text { Ayudante }\end{array}$ & l & 3.28 & 1.447 & 2.72 & 1.526 & 2.94 & 1.765 & 3.33 & 1.328 \\
\hline Ayudante Doctor & & 3.00 & 1.252 & 2.42 & 1.222 & 2.53 & 1.428 & 2.87 & 1.143 \\
\hline Colaborador Licenciado & & 3.94 & 1.124 & 1.75 & .931 & 2.13 & 1.455 & 2.94 & 1.482 \\
\hline Contratado Doctor & & 3.26 & 1.468 & 2.27 & 1.308 & 1.89 & 1.162 & 2.57 & 1.209 \\
\hline Titular / Agregado & & 3.21 & 1.566 & 2.58 & 1.465 & 1.33 & .780 & 2.43 & 1.370 \\
\hline Catedrático & & 3.15 & 1.309 & 2.54 & 1.253 & 1.21 & .732 & 2.23 & 1.038 \\
\hline
\end{tabular}

Nota: Tiempo = Falta de tiempo; Formación = He considerado más importante otro tipo de formación; Docente $=$ Llevo poco tiempo como docente; Ofertas = Falta de ofertas educativas cercanas. 
El estudio de la formación universitaria recibida en relación con los impedimentos, muestra resultados estadísticamente significativos en el caso de, "Ilevo poco tiempo como docente" y "falta de ofertas formativas cercanas" (Tabla 2). En ambos casos esto se observa entre Diplomados y Licenciados $(p<.05)$ e Ingenieros Superiores $(p<.05$; marginalmente significativa en falta de ofertas formativas cercanas). Adicionalmente, también se encuentra en el caso de falta de ofertas formativas cercanas, entre Diplomados y Graduados (Tabla 5).

Tabla 5 .

Estadísticos descriptivos en impedimentos según Formación universitaria recibida

\begin{tabular}{lllllllll}
\hline & \multicolumn{2}{l}{ Tiempo } & \multicolumn{2}{c}{ Formación } & \multicolumn{2}{c}{ Docente } & \multicolumn{2}{c}{ Ofertas } \\
& $M$ & DT & $M$ & DT & $M$ & DT & $M$ & DT \\
\hline Licenciado & 3.50 & 1.249 & 2.78 & 1.166 & 2.89 & 1.641 & 3.72 & 1.320 \\
Graduado & 3.30 & 1.259 & 2.35 & 1.301 & 2.30 & 1.460 & 2.43 & 1.080 \\
Ingeniero superior & 3.24 & 1.443 & 2.34 & 1.314 & 1.93 & 1.306 & 2.64 & 1.335 \\
Diplomado & 3.28 & 1.634 & 2.78 & 1.166 & 1.83 & 1.282 & 2.79 & 1.364 \\
\hline
\end{tabular}

Nota: Tiempo = Falta de tiempo; Formación = He considerado más importante otro tipo de formación; Docente $=$ Llevo poco tiempo como docente; Ofertas = Falta de ofertas educativas cercanas .

Del mismo modo, la formación posterior obtuvo diferencias significativas en, "llevo poco tiempo como docente" y "falta de ofertas formativas cercanas" (Tabla 2). En ambos casos se puede observar la diferencia entre el profesorado que tiene estudios de Máster y Doctorado (Tabla 6).

Tabla 6.

Estadísticos descriptivos en impedimentos según Formación posterior

\begin{tabular}{lllllllll}
\hline & \multicolumn{2}{l}{ Tiempo } & \multicolumn{3}{c}{ Formación } & \multicolumn{2}{c}{ Docente } & \multicolumn{2}{c}{ Ofertas } \\
& $M$ & DT & $M$ & DT & $M$ & DT & $M$ & DT \\
\hline CAP & 3.76 & 1.20 & 2.94 & 1.43 & 2.41 & 1.54 & 3.00 & 1.45 \\
Máster & 3.21 & 1.42 & 2.14 & 1.17 & 2.78 & 1.62 & 3.01 & 1.35 \\
Doctorado & 3.24 & 1.45 & 2.39 & 1.34 & 1.79 & 1.19 & 2.61 & 1.32 \\
\hline
\end{tabular}

Nota: Tiempo = Falta de tiempo; Formación = He considerado más importante otro tipo de formación; Docente $=$ Llevo poco tiempo como docente; Ofertas = Falta de ofertas educativas cercanas .

\section{Discusión y conclusiones}

A la luz de los resultados obtenidos, una primera cuestión que cabe destacar son los impedimentos que se identifican como uno de los principales problemas que impiden que el profesorado. Aspectos como la falta de tiempo, ya fue expuestos por Cabello y Antón (2005) como un problema importante. En este sentido, los autores destacan que, por tanto, parece ser que en la actualidad las iniciativas de formación ofertadas desde las instituciones no 
tienen el efecto deseado a pesar de haber pasado más de una década donde se ha venido insistiendo en la importancia de dicha formación. Es, por esta razón, que Fernández (2008) ya consideraba que el carácter eminentemente voluntario e individual de dichas actuaciones formativas afectaba negativamente a la formación docente en el ámbito universitario.

Con respecto a la falta de ofertas formativas cercanas, trabajos previos (Delgado, 2013; Sánchez y Zubillaga, 2005) revelaban que un alto porcentaje de las acciones formativas se habían materializado en forma de jornadas, cursos o talleres. En esta línea, se ha comprobado que el profesorado con menos edad, experiencia y figura contractual, acusan en mayor medida la falta de dichas ofertas, cuestión que también queda de relevancia en el trabajo realizado por Koc, Demirbilek y Yilmaz (2015). De esta manera, es, por tanto, importante desarrollar planes que tengan en cuenta especialmente a aquellas figuras profesionales dentro de la universidad con mayor riesgo de precariedad. Una posible solución a la que apuntan los autores es la creación de una carrera docente con una etapa de menor peso en la gestión y en la docencia en figuras como los ayudantes doctores para favorecer la posibilidad de realizar formaciones adecuadas y valiosas para su función docente. Sin embargo, lo expuesto anteriormente contrasta con la motivación y predisposición del profesorado para formarse, que, según los resultados encontrados, fue elevada. Tanto es así, que dicha cuestión mostró significatividad sobre la formación universitaria recibida para Licenciados, Diplomados, Ingenieros Superiores y Graduados; y para la formación posterior concretamente entre el profesorado que realizó Máster y Doctorado.

Por otro lado, a la pregunta "considero más importante otro tipo de formación", los datos obtenidos estuvieron por debajo de la media, de lo que se extrae que los participantes valoraron más positivamente la formación en métodos activos que otro tipo de formación. Esto corrobora lo hallado por Buenestado (2019) y Jiménez, González y Tornel (2020) donde, los docentes mostraron actitudes más favorables hacia este tipo de cuestiones. Por este motivo, queda patente que, efectivamente, las metodologías planteadas en las formaciones sí son de calado en el profesor universitario y, por tanto, sería recomendable plantear programas estables que hicieran referencias a ellas en las universidades españolas.

Con respecto al género, las profesoras otorgan una mayor importancia a la formación en métodos activos que los profesores. Cuestión que también ha sido identificado en diversos estudios (Buenestado, 2019, Subbaye y Dhunpath, 2016; Trautwein, 2018; Ulla, 2018), que incluso describen también que las profesoras no solo le dan más importancia, sino que participan de una manera más activa. Los autores de este trabajo realmente no asumen ninguna hipótesis al respecto de esta cuestión.

Al preguntar al profesorado si "estaría dispuesto a seguir formándose sobre métodos activos", un alto porcentaje respondieron que afirmativamente (87,8\%). Esta cuestión evidencia que, una vez que se comienza a trabajar sobre estos métodos, los docentes estarían dispuesto a seguir, pese a los inconvenientes mostrados. Luque y Navarro (2011) también observaron que el profesorado que ya se había obtenido algún tipo de formación similar, estaba dispuesto a continuar con ello, ya que consideraban que la formación didáctica obtenida solía ser escasa para poder implantarla con fiabilidad en sus clases. Por ello, una apuesta clara de las universidades debería ser el proponer actuaciones formativas lo suficientemente completas como para que los docentes dispusieran de las herramientas necesarias para la correcta puesta en funcionamiento en sus respectivas asignaturas. Esto supondría otorgar el peso que le corresponde a la formación didáctica y pedagógica en una etapa donde existe la paradoja de que un docente universitario puede llegar a la máxima categoría profesional en el ámbito educativo sin haber recibido una sola acción formativa en cuestiones de pedagogía, psicología, didáctica o ciencias del comportamiento, entre otras. 
Otra cuestión relevante es la relacionada con las condiciones de las propias formaciones. Trabajos recientes abogan por una formación basada en la evidencia (Larraceleta, 2020). Además, la formación pedagógica del profesorado debe estar suficientemente formalizada en las universidades, debe ser objeto de evaluación sistemática, conllevar incentivos y ser reconocida como méritos para el docente. De esta manera, se considera que un mayor impulso en la formación inicial sobre métodos activos del profesorado en los grados o postgrados que se dirigen a la función docente favorecería la compresión del cambio de rol docente y otorgaría una base en este tipo de formación sin necesidad de que uno de los mayores impedimentos en este aspecto (la falta de tiempo) tenga cabida.

Por todo ello, los autores de este estudio concluyen que existe una necesidad de apostar por un sistema de optimización de las formaciones docentes que se llevan a cabo en las universidades y por el establecimiento de controles que permitan otorgar los incentivos suficientes para que cualquier profesor universitario se motive en la mejora de su calidad docente a través de metodologías activas.

\section{Referencias}

Arribas, J. M., Carabias, D., \& Monreal, I. (2010). La docencia universitaria en la formación inicial del profesorado. El caso de la escuela de magisterio de Segovia. Revista electrónica interuniversitaria de formación del profesorado, 13(3), 27-35.

Buenestado, M. (2019). La formación docente del profesorado universitario. El caso de la universidad de córdoba (diagnóstico y diseño normativo) (Tesis Doctoral). Universidad de Córdoba. Córdoba, España.

Caballero, K. y Bolívar A. (2015). El profesorado universitario como docente: hacia una identidad profesional que integre docencia e investigación. REDU-Revista de Docencia Universitaria, 13(1), 57-77. https://doi.org/10.4995/redu.2015.6446

Cabello, J., \& Antón, P. (2005). Conversaciones con el profesorado. Un estudio en cuatro universidades españolas sobre el Espacio Europeo y el uso de las TIC. Revista de Educación, (337), 149-167.

Cohen, J. (1988). Statistical Power Analysis for the Behavioral Sciences. New York, NY: Routledge Academic

Cohen, J. (1992). A power primer. Psychological Bulletin, 112, 155-159. https://doi.org/10.1037/0033-2909.112.1.155

Comisión Europea. (2017). Agenda Renovada de la Unión Europea para la educación superior. Bruselas: European Commission Press.

Delgado, V. (2013). La formación del profesorado universitario. Análisis de los programas formativos de la Universidad de Burgos (Tesis Doctoral). Universidad de Brugos. Burgos, España.

Domingo-Coscollola, M., Bosco, A., Carrasco Segovia, S., \& Sánchez Valero, J. A. (2020). Fomentando la competencia digital docente en la universidad: Percepción de estudiantes y docentes. Revista de Investigación Educativa, 38(1), 167-182. https://doi.org/10.6018/rie.340551

Escudero, J. M., (2020). Un cambio de paradigma en la formación continuada del profesorado: escenario, significados, procesos y actores. Revista Qurriculum, 33, 97125. https://doi.org/10.25145/j.qurricul.2020.33.06 
Escudero, J. M. \& Portela, A. (2015). La formación continuada del profesorado: políticas, programas, aprendizajes docentes e impacto en la enseñanza y los aprendizajes del alumnado. En M. A. Flores y F. Ilídio (eds.), Formaçao e Trabalho docente (pp. 25-37). Santo Tirso: De Facto Editores.

Fernández, A. (2008). La formación inicial del profesorado universitario: El título de Especialista Universitario en Pedagogía Universitaria de la Universidad Politécnica de Valencia. Revista Interuniversitaria de Formación del Profesorado, 63(22,3), 141-160.

Gallardo, D. (2010). Plan de formación del profesorado Universidad de Extremadura (20092010). Servicio de Orientación y Formación Docente Universidad de Extremadura.

García, L. F. (2020). Praxis del desarrollo profesional del docente en escuelas exitosas del Departamento de Educación de Puerto Rico (Tesis Doctoral). Universidad de Puerto Rico, Rio Piedras, Puerto Rico.

González, F.E., Macías, E., Rodríguez, M., \& Aguilera, J.L. (2009). Prospectiva y evaluación del ejercicio docente de los profesores universitarios como exponente de buena calidad. Revista de universidad y Sociedad del conocimiento, 6(2), 38-48.

Hazelkorn, E. (2015). Rankings and the reshaping of higher education. The battle for world-class excellence ( $2^{\circ}$ edition). Basingstoke: Palgrave Macmillan.

Jiménez, D. (2018). Métodos activos en el sistema universitario actual. Madrid: Dykinson

Jiménez, D., González, J. J. \& Tornel, M. (2020). Metodologías activas en la universidad y su relación con los enfoques de enseñanza. Profesorado, Revista de Currículum y Formación del Profesorado, 24(1), 76-94. https://doi.org/10.30827/profesorado.v24i1.8173

Jiménez, D., González, J.J., \& Tornel, M. (2018). Formación del profesorado universitario en metodologías y su incidencia en el aula. Estudios pedagógicos, 44(3), 157-172. http://dx.doi.org/10.4067/S0718-07052018000300157

Jiménez, D., Tornel, M., \& González, J. J. (2015). Optimización de un cuestionario mediante un método Delphi y una prueba piloto. Opción, 31(4), 637-638.

Koc, M., Demirbilek, M., \& Yilmaz, E. (2015). A needs assessment for academicians' professional development. Education and Science, 40(177), 297-311. https://doi.org/10.15390/EB.2015.2545

Larraceleta, A. (2020). La formación del profesorado sobre el autismo basada en la evidencia: la Instrucción e Intervención Mediada por Pares. Revista Electrónica Interuniversitaria De Formación Del Profesorado, 23(1). https://doi.org/10.6018/reifop.393811

Luque, A. M. \& Navarro, E. (2011). El aprendizaje cooperativo y la enseñanza de la geografía en el marco del EEES. Didáctica Geográfica, (12), 49-72.

Marcelo, C. (2006). Los principios generales de la formación del profesorado. En A. Alías., C. Gil., A. Riscos., M. Varcárcel y E. Vicario (eds.), Encuentro sobre la formación del profesorado universitario (pp. 27-30). Almería: Editorial Universidad de Almería.

Ministerio de Educación y Ciencia (2006). Propuestas para la renovación de las metodologías educativas en la universidad.

Monereo, C. (2013). La investigación en la formación del profesorado universitario: hacia una perspectiva integradora. Infancia y Aprendizaje, 36(3), 281-291. https://doi.org/10.1174/021037013807533052 
Montero, I., \& León, O. G. (2007). A guide for naming research studies in Psychology. International Journal of Clinical and Health Psychology, 7, 847-862.

Ponce Ruiz, D. V., Gómez, Á., Kruscthalia, L., \& Viteri Intriago, D. A. (2019). Modelo de formación multidimencional del Docente universitario en UNIANDESQuevedo con énfasis en la Investigación Científica. Dilemas Contemporáneos: Educación, Política y valores, 6.

Rabadán, J.A., \& Hernández, E. (2012). Renovación pedagógica en la Sociedad del Conocimiento. Nuevos retos para el profesorado universitario. RED-DUSC, Revista de Educación a Distancia. Sección de Docencia Universitaria en la Sociedad del Conocimiento, (6).

Rodríguez, S. (2020). Lights and shadows in the pedagogical training of university faculty in Spain. REDU. Revista de Docencia Universitaria, 18(1), $143-168$. https://doi.org/10.4995/redu.2020.13183

Sánchez, P., \& Zubillaga, A. (2005). Las universidades españolas ante el proceso de convergencia europeo: análisis de las medidas institucionales y acciones de aplicación y coordinación. Revista de educación, (337), 169-187.

Subbaye, R., \& Dhunpath, R. (2016). Early-career academic support at the University of KwaZulu-Natal: towards a scholarship of teaching. Studies in Higher Education, 41(10), 1803-1819. https://doi.org/10.1080/03075079.2016.1221657

Trautwein, C. (2018). Academics' identity development as teachers. Teaching in Higher Education, 23(8), 995-1010. https://doi.org/10.1080/13562517.2018.1449739

Triadó. X. M., Estebanell, M., Márquez, M. D. y del Corral, I. (2014). Identificación del perfil competencial docente en educación superior. Evidencias para la elaboración de programas de formación continua del profesorado universitario. Revista Española de Pedagogía, (257), 55-76.

Ulla, M. B. (2018). In-service Teachers' Training: The Case of University Teachers in Yangon, Myanmar. Australian Journal of Teacher Education, 43(1), 66-77.

Vargas, M. A., \& Arce, A. L. A. (2020). Percepción de un grupo de docentes de educación primaria acerca de la preparación recibida durante su formación universitaria en cuanto al tema de las Matemáticas. Actualidades Investigativas en Educación, 31-31. https://doi.org/10.15517/AIE.V2ol1.39978

Yerovi, M. M., y Riascos, O. O. V. (2019). Las concepciones epistemológicas, pedagógicas y didácticas del mejor profesorado de las universidades acreditadas en Colombia. Folios, (49), 19-40. https://doi.org/10.17227/folios.49-9388 